\title{
Metastatic Infiltrating Bladder Urothelial Carcinoma, Sarcomatoid Variant
}

National Cancer Institute

\section{Source}

National Cancer Institute. Metastatic Infiltrating Bladder Urothelial Carcinoma,

Sarcomatoid Variant. NCI Thesaurus. Code C157766.

Bladder sarcomatoid variant urothelial carcinoma that has spread to another anatomical site. 